\title{
NMR Crystallography in the Enzyme Active Site of Tryptophan Synthase
}

\author{
Leonard J. Mueller
}

Department of Chemistry, University of California, Riverside, 92521

Chemical level details such as protonation and hybridization state are critical for understanding enzyme mechanism and function. Even at high resolution, these details are difficult to determine by X-ray crystallography alone. The chemical shift in nuclear magnetic resonance, however, is an extremely sensitive probe of chemical environment, making solidstate NMR spectroscopy and X-ray crystallography a powerful combination for defining chemically-detailed three dimensional structures. Tying them together is computational chemistry, which allows specific models of the chemical structures to be built upon the coarse $\mathrm{X}$-ray frameworks and then tested by comparisons of predicted and assigned chemical shifts. Here we adopt this synergistic approach to interrogate a carbanionic/quinonoid intermediate analogue in the $\beta$-subunit active site of the PLP-requiring enzyme tryptophan synthase. Most significantly, we find that a deprotonated pyridine nitrogen on PLP precludes formation of a true quinonoid species and that there is an equilibrium between the phenolic and protonated Schiff base tautomeric forms. Natural bond orbital analysis indicates that the latter builds up negative charge at the substrate $\mathrm{C}^{\alpha}$ and positive charge at $\mathrm{C}^{\prime}$ ' of the cofactor, consistent with its role as the catalytic tautomer. These findings support the hypothesis that the specificity for $\beta$-elimination/replacement vs. transamination is dictated in part by the protonation states of ionizable groups on PLP and the reacting substrates and underscore the essential role that NMR crystallography can play in characterizing both chemical structure and dynamics within functioning enzyme active sites.
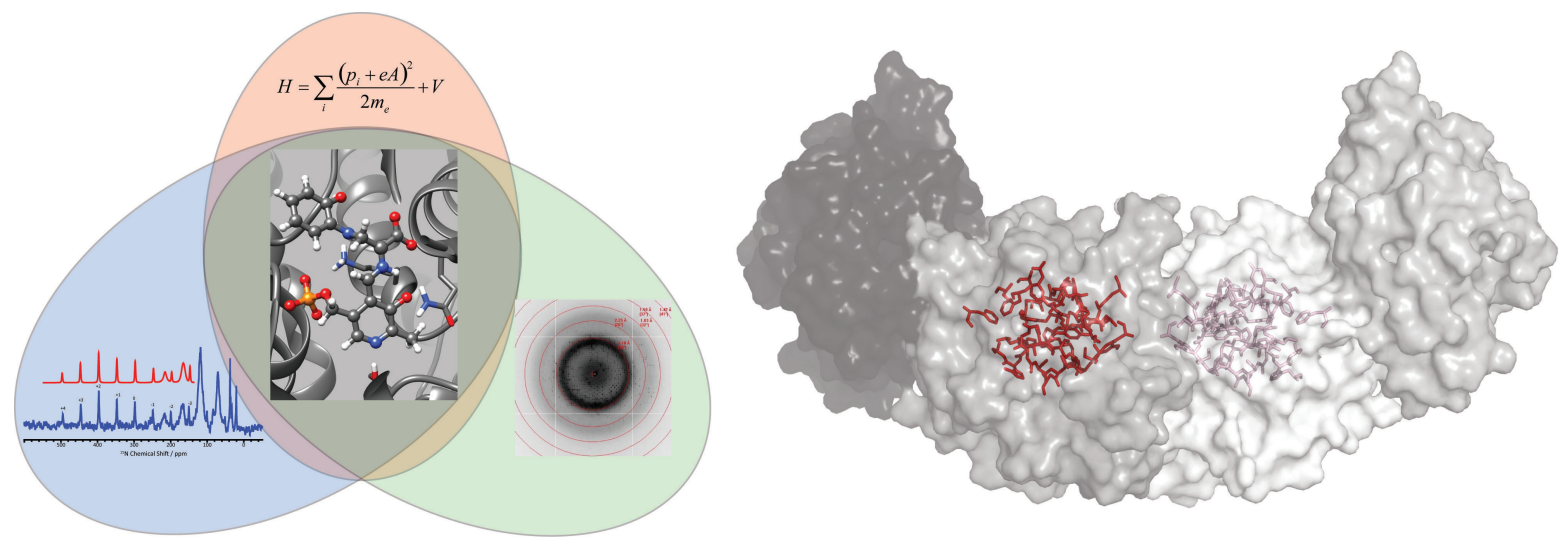

\section{References}

Caulkins et al, Journal of the American Chemical Society, 138, 15214-15226 (2016)

Mueller and Dunn, Accounts of Chemical Research, 46, 2008-2017 (2013)

Young et al, Angewandte Chemie International Edition, 55, 1350-1354 (2016)

Hartman et al, Journal of Biological NMR, 62, 327-340 (2015)

Caulkins et al, Journal of the American Chemical Society, 136, 12824-12827 (2014) 